\title{
Fecal Strings Associated with Streptococcus agalactiae Infection in Nile Tilapia, Oreochromis niloticus
}

\author{
David J. Pasnik ${ }^{*}, 1$, Joyce J. Evans ${ }^{1}$ and Phillip H. Klesius ${ }^{2}$ \\ ${ }^{1}$ Aquatic Animal Health Research Laboratory (AAHRL), United States Department of Agriculture/Agricultural Research \\ Service (USDA/ARS), Chestertown, MD 21620, USA \\ ${ }^{2}$ AAHRL, USDA/ARS, Auburn, AL 38756, USA
}

\begin{abstract}
This study provides the first description of long fecal strings in Streptococcus agalactiae-infected fish. Nile tilapia (Oreochromis niloticus) were administered an intraperitoneal injection with $0.1 \mathrm{~mL}$ solution containing a 10 -fold dilution from $10^{1}$ to $10^{6} \mathrm{cfu} S$. agalactiae/fish. While infected fish developed clinical signs commonly associated with $S$. agalactiae infection, up to $40 \%$ of infected fish in each group also produced considerably longer (up to $20 \mathrm{~cm}$ in length) fecal waste strings than unchallenged tilapia. Fecal strings from these infected fish were observed trailing from the anus and often became increasingly opaque and white over time. Though $S$. agalactiae was not isolated from the fecal strings themselves, all $S$. agalactiae-challenged fish sampled were culture-positive. Histologic examination of the intestines of infected fish exhibited the presence of sloughed intestinal mucous cells and enterocytes in the feces and the absence of normal fecal matter. The presence of long brown and/or white, opaque fecal strings may suggest a clinical sign of bacterial disease and the need for further clinical examination.
\end{abstract}

Streptococcus agalactiae affects numerous wild and cultured fish species worldwide and causes disease involving septicemia and colonization of numerous organs [1-4]. Clinical signs of $S$. agalactiae infection include lethargy or excitability, going off feed, ' $\mathrm{C}$ '-shaped body posturing, erratic swimming and whirling, opercular clearing, spinal curvature, and death $[2,3,5]$. Nile tilapia (Oreochromis niloticus) are susceptible to $S$. agalactiae infection [3-5]. In a variety of previous $S$. agalactiae challenge studies at the Aquatic Animal Health Research Laboratory, Nile tilapia $(n>500$; weight ranging from 3 to $100 \mathrm{~g}$ ) were injected intraperitoneally with doses ranging from $10^{1}$ to $10^{9}$ colony-forming units (cfu) S. agalactiae/fish and maintained at approximately $30^{\circ} \mathrm{C}$. Though these studies were not intended to exclusively examine $S$. agalactiae-related clinical signs, it was nonetheless noted that numerous challenged fish produced long fecal strings. Since fecal strings have not been previously associated with $S$. agalactiae infection, a study was performed to examine the presence of fecal strings after experimental $S$. agalactiae challenge.

Nile tilapia (O. niloticus) with a mean weight of $13.9 \pm$ $0.6 \mathrm{~g}$ were housed at the USDA/ARS Aquatic Animal Health Laboratory in Chestertown, Maryland, USA. The fish were kept in $57 \mathrm{~L}$ glass aquaria supplied with flow-through dechlorinated tap water, maintained at a mean temperature of $30.5 \pm 0.6^{\circ} \mathrm{C}$, mean dissolved oxygen of $5.3 \pm 0.9 \mathrm{mg} / \mathrm{L}$, and mean ammonia concentration of $0.2 \pm 0.8 \mathrm{mg} / \mathrm{L}$. The fish were also fed daily to satiation with Aquamax feed (Brentwood, Missouri, USA) and maintained on a $12 \mathrm{~h} \mathrm{:} 12 \mathrm{~h}$ light :

*Address correspondence to this author at the Aquatic Animal Health Research Laboratory, 118B Lynchburg Street, United States Department of Agriculture/Agricultural Research Service, Chestertown, Maryland 21620, USA; Tel: 410-778-4136; E-mail: david.pasnik@ars.usda.gov dark period. Tilapia were challenged with an $S$. agalactiae isolate obtained from a mullet, Liza klunzingeri, from $\mathrm{Ku}-$ wait Bay, Kuwait [3]. The isolate was grown at $30^{\circ} \mathrm{C}$ for 24 $\mathrm{h}$ on $5 \%$ sheep blood agar (SBA; Remel, Lenexa, Kansas, USA) before reconstitution in tryptic soy broth (TSB; Remel) to create the challenge solution. Tilapia were administered an intraperitoneal injection with $0.1 \mathrm{~mL}$ solution containing a 10 -fold dilution from $10^{1}$ to $10^{6} \mathrm{cfu} S$. agalactiae/fish. Ten fish were used for each dose and sequestered into separate tanks accordingly. Ten control fish were injected with TSB. The intraperitoneal challenge route was utilized because it is a reproducible and reliable method ensuring challenge of all individual fish with a uniform bacterial dose $[6,7]$.

Fish were monitored for 7 days post-challenge; clinical signs of disease were recorded once daily while mortalities were removed three times daily. Swab samples were aseptically obtained from the nares, brain, anterior kidney, and posterior intestine of $20 \%$ of dead fish from each group and cultured overnight on SBA at $30^{\circ} \mathrm{C}$ to confirm the presence of $S$. agalactiae. In order to confirm isolate identity as $S$. agalactiae, two bacterial isolates obtained from the brains of 2 challenged fish were tested with the BIOLOG Mi$\operatorname{croLog} 3^{\mathrm{TM}}$ system according to the manufacturer's instructions (BIOLOG Inc., Hayward, California, USA) [3]. For histologic examination, intestinal tissues from 10 fish in the challenge groups and 5 fish from the control group were sampled 4 days post-challenge. Samples were fixed in $10 \%$ neutral buffered formalin, embedded in paraffin, sectioned at $5 \mu \mathrm{m}$, and stained with Harris' hematoxylin and eosin. Any moribund fish in the study were humanely euthanized with an overdose of tricaine methanesulfonate (MS-222; Argent Chemical Laboratories, Redmond, Washington, USA). 
Within 7 days post-challenge, fish in each group developed overt clinical signs of disease, such as going off feed, lethargy, bilateral exophthalmia, erratic swimming, and death. Mortalities within each challenge group began 1 day post-challenge and most had occurred by 3 days postchallenge. Several of the $S$. agalactiae-infected tilapia produced considerably longer (up to $20 \mathrm{~cm}$ in length) fecal waste strings (Fig. 1, Table 1) than unchallenged tilapia, irrespective of weight or challenge dose. Strings were observed trailing from the anus and often became increasingly opaque and white as time increased. Long but brown fecal strings started to appear within 2 days post-challenge and began to appear as white, mucoid fecal strings within 4 days postchallenge. At this latter point, the fecal strings would contain intermittent sections of white, presumptively mucoid material and brown feces or the whole fecal strings would contain entirely white, presumptively mucoid material devoid of brown feces.

Given that fish were not individually identified, the numbers of fish per group showing certain clinical signs was determined as the maximum number observed exhibiting a clinical sign in one of the seven post-challenge days. Note that this may ultimately underestimate the total number of fish that showed a clinical sign if they stop exhibiting a clinical sign from one day to the next. When comparing maximum numbers of fish showing a certain clinical sign in one day with the total overall number of fish challenged ( 60 fish), the percentage of fish showing a specific clinical sign was: mortalities $(53 \%)$, confined to tank bottom (43\%), lethargy (32\%), fecal strings (28\%), stationary $(25 \%)$, slow or no feeding response $(18 \%)$, darkened coloration $(12 \%)$, and serpentine swimming (2\%). Within each challenge dose group, the maximum percentage of fish to have fecal strings in one day was 20 to $40 \%$ and a higher percentage did not appear to be related to dose.

Samples for bacteriology obtained from the nares, brain, anterior kidney, and posterior intestines were all positive for bacterial growth. Bacterial cultures from all sampled organs elicited primarily smooth, white, circular, Gram-positive, oxidase-negative colonies, and isolates were identified as $S$. agalactiae with the BIOLOG system (Probability $=98$ to 99\%). Control fish injected with TSB did not die or exhibit clinical signs of disease (including no fecal strings) and were negative for $S$. agalactiae growth. Light microscopic examination of intestinal sections from ten challenged fish did not reveal severe or consistent alterations. Two fish had food in the intestinal tract, and two fish exhibited focal areas of moderately increasing mucus cell numbers in the anterior intestine. Three fish showed mild inflammatory cell infiltration in the submucosa, and one fish exhibited mild focal enterocyte necrosis with sloughing of mucosal cells. No significant changes were noted among five control fish when compared to normal tilapia intestinal histologic sections [8].

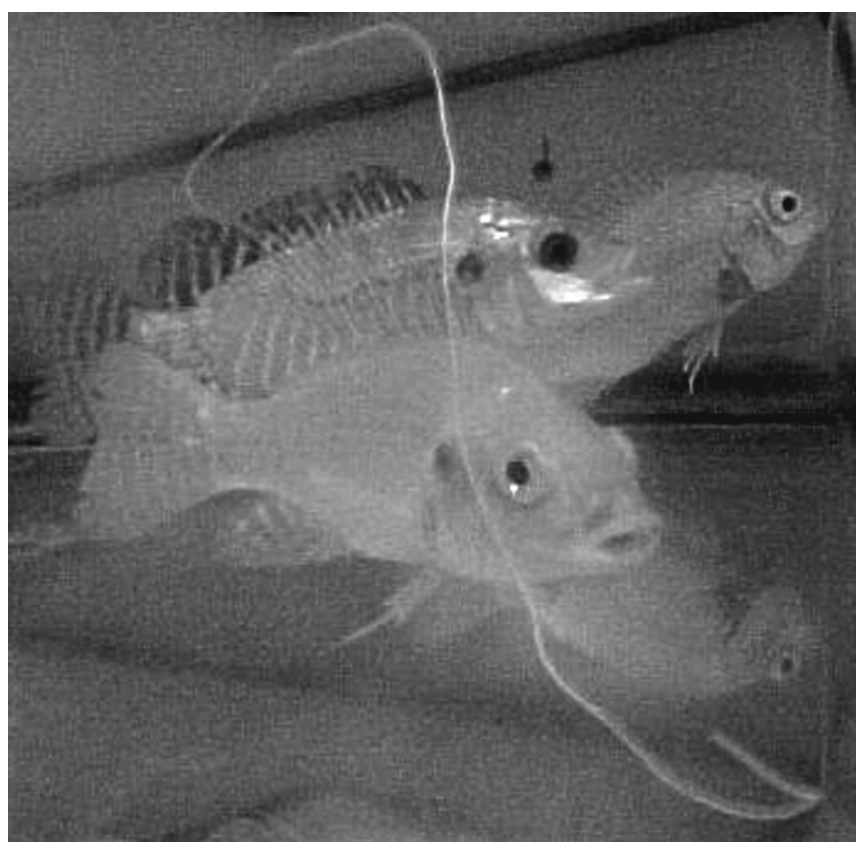

Fig. (1). Nile tilapia (Oreochromis niloticus) challenged with Streptococcus agalactiae exhibiting a long, white mucoid fecal string trailing from the anus.

Long mucoid fecal strings in S. agalactiae-infected fish have also not been previously described, but long fecal

Table 1. Percentage of 10 Nile Tilapia (Oreochromis niloticus) in Each Dose Group Exhibiting Clinical Signs After Challenge with Streptococcus agalactiae ${ }^{1}$

\begin{tabular}{|c|c|c|c|c|c|c|c|c|}
\hline \multirow{2}{*}{ Dose (cfu/Fish) } & \multicolumn{8}{|c|}{ Clinical Sign } \\
\hline & Mortalities & $\begin{array}{c}\text { Confined to } \\
\text { Tank Bottom }\end{array}$ & Lethargy & $\begin{array}{l}\text { Fecal } \\
\text { Strings }\end{array}$ & Stationary & $\begin{array}{l}\text { Slow or No Feed- } \\
\text { ing Response }\end{array}$ & $\begin{array}{l}\text { Darkened } \\
\text { Coloration }\end{array}$ & $\begin{array}{l}\text { Serpentine } \\
\text { Swimming }\end{array}$ \\
\hline Control & 0 & 0 & 0 & 0 & 0 & 0 & 0 & 0 \\
\hline $10^{1}$ & 70 & 70 & 30 & 30 & 20 & 20 & 0 & 10 \\
\hline $10^{2}$ & 60 & 50 & 30 & 30 & 10 & 20 & 10 & 0 \\
\hline $10^{3}$ & 70 & 30 & 20 & 30 & 10 & 30 & 10 & 0 \\
\hline $10^{4}$ & 30 & 30 & 30 & 20 & 30 & 20 & 10 & 0 \\
\hline $10^{5}$ & 70 & 30 & 30 & 40 & 30 & 10 & 10 & 0 \\
\hline $10^{6}$ & 20 & 50 & 50 & 20 & 50 & 10 & 30 & 0 \\
\hline Overall Percent $^{2}$ & 53 & 43 & 32 & 28 & 25 & 18 & 12 & 2 \\
\hline
\end{tabular}

${ }^{1}$ Tilapia were administered an intraperitoneal injection with $0.1 \mathrm{~mL}$ inoculum containing a 10 -fold dilution from $10^{1}$ to $10^{6}$ colony-forming units (cfu) $S$. agalactiae/fish. Ten fish were used for each dose and sequestered into separate tanks accordingly. Ten control fish were inoculated with TSB. Fish were monitored daily for clinical signs of disease and mortality for $7 \mathrm{~d}$ post-challenge.

${ }^{2}$ Overall percent is determined by the number of fish exhibiting a clinical sign per all 60 fish challenged. 
strings were commonly observed in individual infected fish in our challenge studies. The feces were not cultured due to the high probability of contamination with water-borne $S$. agalactiae. While $S$. agalactiae was not isolated from the fecal strings themselves, fecal strings were only observed coming from $S$. agalactiae-challenged fish and not controls, all $S$. agalactiae-challenged fish sampled were culturepositive (including all posterior intestine samples), and all control fish were culture-negative. This suggests that $S$. agalactiae-challenged fish developed systemic infections and that the resulting disease included fecal string production. They resembled feces termed "mucoid fecal casts" described in select fish viral diseases, presumably created due to the 1) presence of sloughed intestinal mucous cells and enterocytes in the feces and 2) absence of normal fecal matter. This composition of the fecal strings are suggested by other publications [9-11] and by histologic examination of the intestinal tissue of the challenged fish. Fecal strings are not frequently examined or emphasized in relation to fish disease, though strings presumably containing sloughed intestinal mucosa and infectious organisms have been observed with a number of viral fish pathogens: Infectious Pancreatic Necrosis Virus, Infectious Hematopoietic Necrosis Virus, and Salmonid Herpesvirus Type 1 in salmonids and Spring Viremia of Carp [10, 11]. However, fecal strings are not routinely associated with bacterial diseases in fish. The presence of fecal strings can be cause for concern, suggesting a clinical sign of viral or bacterial disease and the need for further etiological examination. Fecal analysis is commonly used for diagnosis of bacterial and parasitic infections in mammals [12], but is not so common in aquatic animal medicine. Fish feces have been collected in studies that measure parameters such as: intestinal flora composition [13], feed consumption and digestibility [14], and endocrine activity [15]. However, clinical fecal analysis and observation could add to the battery of non-invasive diagnostic tests for viral and now bacterial diseases available to the aquatic animal clinician. Such clinical tests may be helpful because they can indicate disease presence, though they may not be as useful in acute disease. In this study, acutely affected fish died before the appearance of fecal strings. Fecal strings in the tanks may also promote $S$. agalactiae fecal-oral transmission, because streptococcal infections are known to occur after ingestion of materials containing streptococcal organisms $[16,17]$. Fecesrelated clinical signs and tests may be more useful for tilapia or other fish species maintained in clearer water (i.e. laboratory or aquarium tanks) than murkier water (i.e. ponds), because the strings can be readily observed as the fish swim. But these fecal strings have also been noted clinging to fish removed from water and may be analyzed even if the fish cannot be directly observed swimming with trailing fecal strings.

\section{ACKNOWLEDGEMENTS}

The authors thank Daniel Brougher for his technical assistance. This work was supported under USDA/ARS CRIS Number 6420-32000-022-00D. All research was approved by the USDA/ARS AAHRL Institutional Animal Care and Use Committee. Mention of trade names or commercial products does not imply recommendation by the USDA.

\section{REFERENCES}

[1] Robinson JA, Meyer FP. Streptococcal fish pathogen. J Bacteriol 1966; 92(2): 512.

[2] Baya AM, Lupiani B, Hetrick FM, et al. Association of Streptococcus sp. with fish mortalities in the Chesapeake Bay and its tributaries. J Fish Dis 1990; 13: 251-3.

[3] Evans JJ, Klesius PH, Glibert PM, et al. Characterization of betahaemolytic Group B Streptococcus agalactiae in cultured seabream, Sparus auratus L., and wild mullet, Liza klunzingeri (Day), in Kuwait. J Fish Dis 2002; 25(9): 505-13.

[4] Evans JJ, Klesius PH, Shoemaker CA. An overview of Streptococcus in warmwater fish. Aquacult Health Int 2006; 7: 10-14.

[5] Pasnik DJ, Evans JJ, Klesius PH. Development of skeletal deformities in a Streptococcus agalactiae-challenged male Nile tilapia (Oreochromis niloticus) broodfish and in its offspring. Bull Eur Assoc Fish Pathol 2007; 27(5): 169-76.

[6] Nordmo R. In: Gudding R, Lillehaug A, Midtlyng PJ, et al. Eds. Fish vaccinology: Developments in biological standardization, Karger, Basel, Switzerland, 1997; 90: 303-9.

[7] Nordmo R, Ramstad A. Comparison of different challenge methods to evaluate the efficacy of furunculosis vaccines in Atlantic salmon (Salmo salar L.). J Fish Dis 1997; 20: 119-126.

[8] Morrison CM, Fitzsimmons K, Wright Jr. JR. Atlas of tilapia histology; Baton Rouge, LA: World Aquaculture Society; 2006.

[9] Yasutake WT. In: Ribelin WE, Migaki G. Eds. The pathology of fishes, The University of Wisconsin Press, Madison, Wisconsin, 1975.

[10] Plumb JA. Health maintenance and principal microbial diseases of cultured fishes; Ames, Iowa: Iowa State University Press; 1999.

[11] Noga EJ. Fish disease: Diagnosis and treatment; Ames, Iowa: Iowa State University Press; 2000.

[12] Kahn CM, Line S, Aiello SE. Eds. The Merck veterinary manual. 9th Ed.; Philadelphia, Pennsylvania: National Publishing, Inc.; 2005.

[13] Sugita H, Miyajima C, Kobiki Y, Deguchi Y. The daily fluctuation and inter-individual variation of the faecal flora of carp, Cyprinus carpio L. J Fish Biol 1990; 36(1): 103-105.

[14] Sugiura S, Dong F, Hardy R. Primary responses of rainbow trout to dietary phosphorus concentrations. Aquacult Nutr 2000; 6(4): 23545.

[15] Turner JW Jr, Nemeth R, Rogers C. Measurement of fecal glucocorticoids in parrotfishes to assess stress. Gen Comp Endocrinol 2003; 133: 341-52.

[16] Minami T, Nakamura M, Ikeda Y, Ozaki H. A beta-hemolytic Streptococcus isolated from cultured yellowtail. Fish Pathol 1979; 14(1): 33-8.

[17] Bromage ES, Owens L. Infection of barramundi Lates calcarifer with Streptococcus iniae: Effects of different routes of exposure. Dis Aquat Org 2002; 52(3): 199-205. 